\title{
Space Bioprocess Engineering on the Horizon
}

\author{
Aaron J. Berliner ${ }^{1,2, *}$, Isaac Lipsky ${ }^{1,2}$, Davian Ho ${ }^{1,2}$, Jacob M. Hilzinger ${ }^{1,2}$, Gretchen \\ Vengerova $^{1,2}$, George Makrygiorgos ${ }^{1,3}$, Matthew J. McNulty ${ }^{1,4}$, Kevin Yates ${ }^{1,4}$, Nils J.H. \\ Averesch $^{1,5}$, Charles S. Cockell ${ }^{1,6}$, Tyler Wallentine ${ }^{1,7}$, Lance C. Seefeldtt ${ }^{1,7}$, Craig S. \\ Criddle $^{1,5}$, Somen Nandi ${ }^{1,4,8}$, Karen A. McDonald ${ }^{1,4}$, Amor A. Menezes ${ }^{1,9}$, Ali Mesbah ${ }^{1,3}$, \\ and Adam P. Arkin ${ }^{1,2, *}$
}

\author{
${ }^{1}$ Center for the Utilization of Biological Engineering in Space (CUBES), http: / / cubes . space / \\ ${ }^{2}$ Department of Bioengineering, University of California Berkeley, Berkeley, CA \\ ${ }^{3}$ Department of Chemical and Biomolecular Engineering, University of California Berkeley, Berkeley, CA \\ ${ }^{4}$ Department of Chemical Engineering, University of California, Davis, Davis, CA \\ ${ }^{5}$ Department of Civil and Environmental Engineering, Stanford University, Stanford, CA \\ ${ }^{6}$ UK Centre for Astrobiology, School of Physics and Astronomy, University of Edinburgh, Edinburgh, UK \\ ${ }^{7}$ Department of Chemistry and Biochemistry, Utah State University, Logan, UT \\ ${ }^{8}$ Global HealthShare Initiative, Davis, CA \\ ${ }^{9}$ Department of Mechanical and Aerospace Engineering, University of Florida, Gainesville, FL \\ *Corresponding authors e-mail: aaron.berliner@berkeley.edu and aparkin@lbl.gov
}

Reinvigorated public interest in human space exploration has led to the need to address the science and engineering challenges described by NASA's Space Technology Grand Challenges (STGCs) for expanding the human presence in space. Here we define Space Bioprocess Engineering (SBE) as a multi-disciplinary approach to design, realize, and manage a biologically-driven space mission as it relates to addressing the STGCs for advancing technologies to support the nutritional, medical, and incidental material requirements that will sustain astronauts against the harsh conditions of interplanetary transit and habitation offworld. SBE combines synthetic biology and bioprocess engineering under extreme constraints to enable and sustain a biological presence in space. Here we argue that SBE is a critical strategic area enabling long-term human space exploration; specify the metrics and methods that guide SBE technology life-cycle and development; map an approach by which SBE technologies are matured on offworld testing platforms; and suggest a means to train the next generation spacefaring workforce on the SBE advantages and capabilities. In doing so, we outline aspects of the upcoming technical and policy hurdles to support space biomanufacturing and biotechnology. We outline a perspective marriage between space-based performance metrics and the synthetic biology Design-Build-Test-Learn cycle as they relate to advancing the readiness of SBE technologies. We call for a concerted effort to ensure the timely development of SBE to support long-term crewed missions using mission plans that are currently on the horizon.

Keywords: space systems bioengineering, biomanufacturing, space bioprocess engineering, biotransformation human exploration, in situ resource utilization, life support systems, biomanufacturing, space policy

Biotechnologies may have mass, power and volume advantages compared to abiotic approaches for critical mission elements for long-term crewed space exploration ${ }^{1,2}$. While there has been point progress in demonstration and evaluation of these benefits for specific examples in this field such as for food production, waste recycling, etc., there is only just emerging possible consensus on the scope of the application of biosynthetic and biotransformative technologies to space exploration and there is almost no formal definition of the scope, performance needs and metrics, and technology development cycle for these systems. It is time to formally establish the field of Space Bioprocess Engineering (SBE) to build this nascent community, train the workforce and develop the critical technologies for planned deep-space missions. The inter-sectional nature of SBE (Fig. 1a) implies that the field borrows many elements from a number of related fields such as the synthetic biology design process from Bioengineering, astronaut sustainability ${ }^{3,4}$ and mission design from Astronautics ${ }^{5,6}$, environmental-context and constraints from the Space Sciences, and living systems habitability and distribution concepts from Astrobiology ${ }^{7}$. SBE represents an extension of the standard astronautics paradigm in meeting NASA's Space Technology Grand Challenges (STGCs) for expanding the human presence in space, managing resources in space, and enabling transformative space exploration and scientific discovery ${ }^{8,9}$ (Fig. 1b). Aspirational realizations of SBE would feature prominently in establishment of in-orbit test-facilities, interplanetary waystations, lunar habitats, and a biomanufactory on the surface of Mars ${ }^{10}$. Differentiated from traditional efforts in space systems engineering, these systems would encapsulate elements from in situ resource utilization 

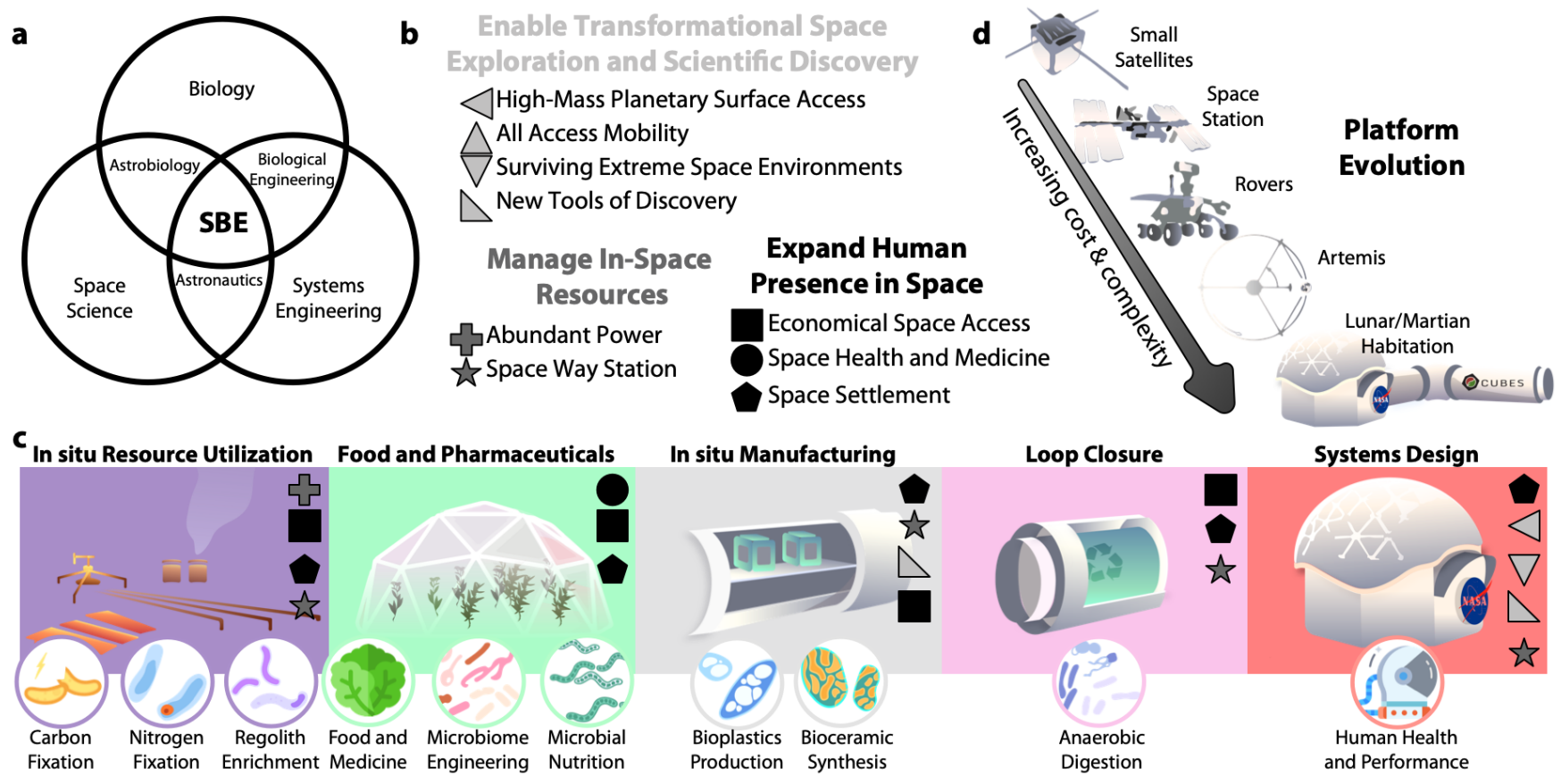

Figure 1. (a) Venn Diagram-based definition of Space Bioprocess Engineering (SBE) as an interdisciplinary field. (b) NASA's space technology grand challenges ${ }^{8}$ key by shape and colored by group. (c) Possible SBE components separated by colors for in situ resource utilization (ISRU), food and pharmaceutical synthesis (FPS), in situ manufacturing (ISM), and loop closure (LC), with the biological processes inherent to each represented below in circles. (d) Platform evolution for biological experiments starting with Earth-orbit CubeSats and proceeding through the ISS, Mars-and-Luna-based rovers, to Lunar and cis-Lunar based human and autonomous systems via the Artemis program.

(ISRU) for the production of biological feedstocks such as fixed carbon and nitrogen for use as inputs for plant and microbial production systems ${ }^{11,12}$, fertilizers for downstream use by plants ${ }^{13}$; in situ (bio)manufacturing (ISM) to produce materials requisite to forge useful tools and replacement parts $^{14}$, food and pharmaceutical synthesis (FPS) via plant and microbial engineering for increased productivity and resilience in space conditions, production of nutrients and protective/therapeutic agents for sustaining healthy astronauts ${ }^{15,16}$; and life-support loop closure (LC) for minimizing waste and regenerating life-support functions and biomanufacturing. Maximizing the productivity of the biomanufacturing elements increases the delivery-independent operating time of a biofoundry in space while minimizing cost and risk. ${ }^{17}$ (Fig. 1c). Ultimately, efforts must be mounted to update the mandate to include SBE as a tool for enabling human exploration; specialize the metrics and methods that guide SBE technology life-cycle and development; further develop means by which SBE technologies are designed for ground testing and matured on offworld testing platforms (Fig. 1d); and train the minds that enter the spacefaring workforce on the SBE advantages and capabilities.

\section{An Inclusive Mandate To Leverage SBE}

While previous strategic surveys such as NASA's Journey to Mars program ${ }^{18}$ the 2018 Biological and Physical Sciences (BPS) Decadal Survey ${ }^{19}$ have acknowledged that plants and microbes may be integral parts of life support and recycling systems but can present challenges to the environmental operation of engineering systems in space due to contamination and other inherent drawbacks. However, none of these have coherently called for the development of the science and technology to engineer these organisms and their biotransformative processes in support of space exploration. The SBE community requires a mandate that identifies mission designs and elements for which engineering biosystems would be most appropriate, and defines the productivity, risk and efficiency targets for these systems in integrated context with other mission elements and in fair comparison to abiotic approaches. This will require integration of SBE resources and knowledge across government, industry, and academia. Previous biological strategies should now specifically call for (1) definition of the physical engineering constraints on the production systems and development of optimized reactor/processing systems for these elements; (2) quantitative assessment of the bioengineering required to meet performance goals in space given the special physiology required in an offworld environment; and (3) development of efficient tooling for offworld genetic engineering along with the proper 
containment and clean-up protocols.

Such a mandate would result in: (1) a deeper, more mechanistic understanding of the growth and phenotypic characteristics of organisms operating in space-based bioprocesses taking into account issues of differences in gravity, radiation, light, water quality, etc.; new applications of these organisms off-planet; (3) new reactors, bioprocess control designs and product processing/delivery technologies accounting for these conditions and the specific constraints of scaling and operational simplicity in space. The development of open, publicly accessible data and tools would enable rigorous comparison among biotechnologies and with abiotic (physical and chemical) approaches within better defined mission-scenarios. Ideally, this should create interative sub-communities that may collaborate and compete on different approaches to meet bioengineering goals and metricize results against the mission specifications.

SBE is an emerging engineering discipline and there are long but feasible routes from discovery, through invention to application. Furthermore, SBE is multidisciplinary and its utility within the larger space community demands specialized cross-training of diverse teams. It in such situations agencies like the Department of Energy (DOE) have found it effective to ensure there is specific funding to support longer term team science to accomplish ambitious scientific and technical goals. The Industrial Assessment Centers (IACs) program is one longest-running DOE programs (started in 1976) and has provided nearly 20,000 no-cost assessments for small- and medium-sized manufacturers and more than 147,000 recommendations in an effort to reduce greenhouse gas emissions without compromising U.S. manufacturing's competitive edge globally ${ }^{20}$. Conversely, successful examples for demonstrating the effect of fostering multidisciplinary centers for space-based biotechnology can be found in NASA's Center for the Utilization of Biological Engineering in Space (CUBES, https: / / cubes . space/), or ESA's Micro-Ecological Life Support System Alternative (MELiSSA, https : / /www. melissafoundation.org/) program - with the capabilities to design, prototype, and ultimately translate biological technologies to space while training the necessary workforce. Such centers are tasked with the development of initial concept trade studies; defining requirements; managing life-support interfaces; evaluating ground integration, operations, and maintenance; coordinating mission operations; and supporting and sustaining engineering and logistics ${ }^{21,22}$. However, these programs are generally restricted to shorter operation timelines - and would benefit from a longer horizon. This is especially true for SBE as biological developments generally require a longer timeframe for integration in industrial endeavors.

\section{Specialization of SBE Metrics and Methods}

Response to the proposed expanded mandate above requires careful consideration of the space-specific performance metrics that SBE must fulfill. Payload volume, mass, and power requirements are made as small as possible and are limited in envelope by their carrier system. One of the most compelling aspects of biotechnology is the ability of such systems to adapt to these constraints relative to certain industrial alternatives. To efficiently evaluate and deploy novel biotechnologies, SBE experiments should begin with standardized unit operations that clearly define the desired biological function. This allows for a standardized experimental framework to test modular biotechnologies not only within the system to be engineered, but also within and between research groups. To define the minimal basis set of unit operations for a given mission, test and optimize the biotechnologies for each unit operation, and integrate each unit operation into a stable system, we adopt the methods from standard bioengineering in the form of a Design-Build-Test-Learn (DBTL) cycle $^{23}$ (Fig. 2).

\section{Performance Metrics}

The design phase of the DBTL cycle begins with the establishment of core constraints and engineering targets that can be explored by standardizing the high-priority performance metrics (\{Modularity, Recyclability, Supportability, Autonomy, Sustainability \})- which we argue gain special weight in space- from which downstream technoeconomic and life-cycle analysis decisions can be explored (Fig. 2a). The space-specific constraints on performance include: (1) an exceptionally strong weighting on a low mass/volume/power footprint for the integrated bioprocess; (2) limited logistic supply of materials and a narrow band of specifically chosen feedstocks; (3) added emphasis on simplicity of set-up, operation and autonomous function to free up astronaut time; (4) mission-context de-risking against cascading failure; (5) strong requirements for efficiency and closed-loop function to maximize efficient resource use and minimize waste products; (5) a critical need for modularity and 'maintainability' so that parts can be swapped easily, new functions added easily, and repairs can be done without logistical support beyond the crew; (6) an increased dependence on other mission elements such as provision of water, gases, astronaut wastes, power, and other raw materials such a regolith which may vary in abundance, quality, and composition in unpredictable ways; (7) the need to design sustainable and supportable operation across long time horizons without logistical support beyond the bounds of the local mission; (8) increased ability to operate in more extreme environments including low gravity, high radiation, low nutrient input, and other stressors; and (9) and process compatibility among common media and operational modes to allow for easy process integration and risk-reduction through redundancy of systems.

Ideally, this combination of performance metrics provides informative constraints on biology and technology choicesd. Feedstock, loop-closure, environmental parameters and product needs will constrain the minimal set of organisms to develop 


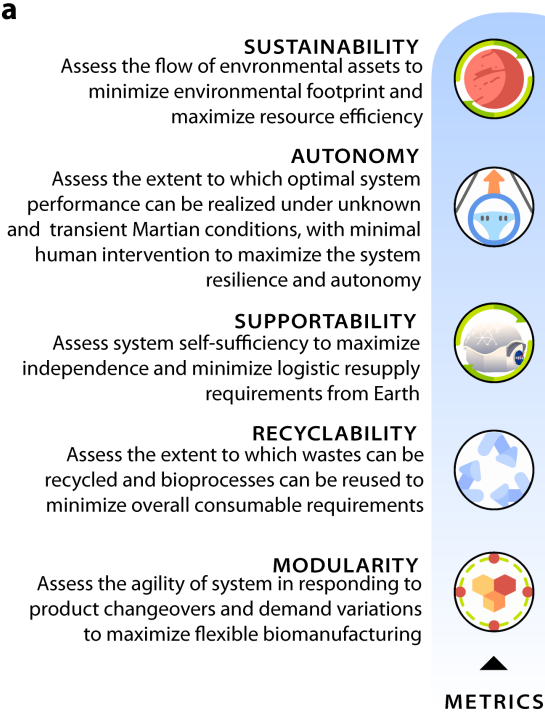

b

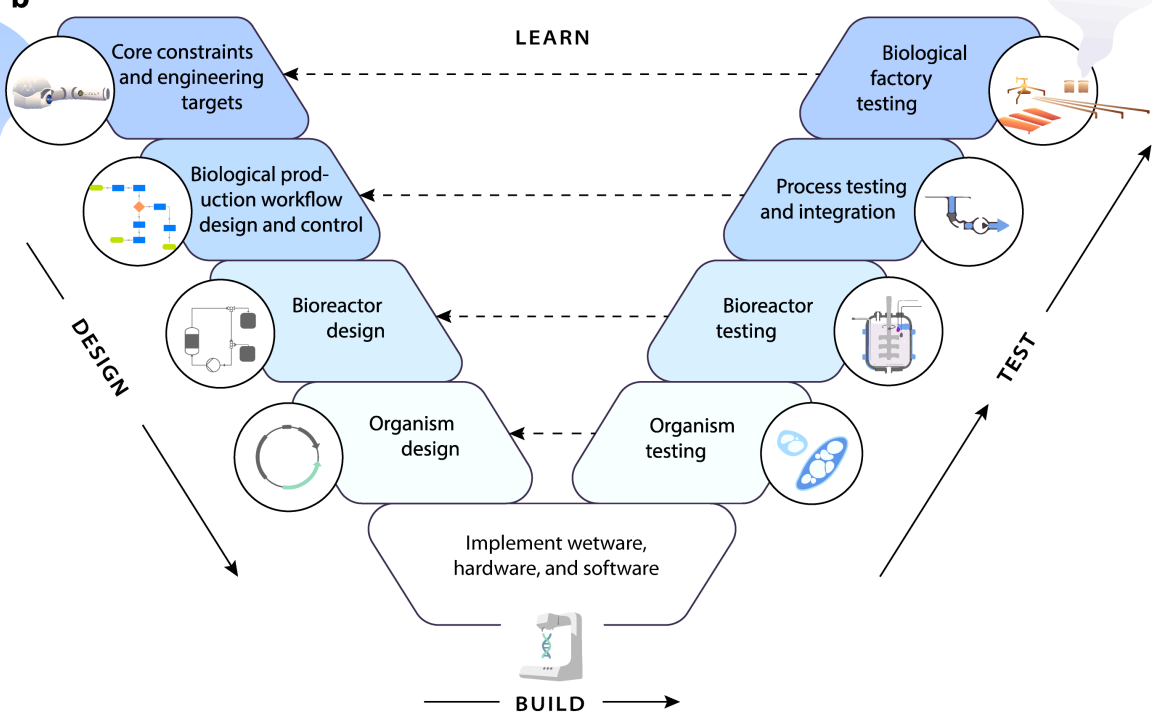

Figure 2. Overview of (a) space systems bioengineering (SBE) performance metrics as core constraints and engineering targets within the (b) diagram of SBE-specific Design, Build, Test, Learn (DBTL) cycle.

and test for growth rate, optimal cultivation, robustness and resilience to space conditions and shelf-life, safety and genetic tractability, product yield, titer and rate, feedstock utilization and waste streams ${ }^{24}$. Once suitable chassis organisms have been evaluated and selected, the DBTL cycle can integrate staged co-design of the optimal process hardware (e.g. molecular biological set-ups, genetic engineering tools, bioreactors, and product post-processing systems) configuration, operating parameters, and process controllers. Operation of the cycle over increasing scale and ever more realistic deployment environments permits controlled traversal of the technology readiness levels for each technology and mission.

\section{Design-Build-Test-Learn}

In the design phase, we argue that efforts must be made to (1) create a database of engineering targets (products, production rates, production yields, production titers, risk factors, waste/recyclability factors, material costs, operational costs, weight, power demand/generation) that set the core constraints for workflow and mission optimization; (2) leverage emerging pathway design software and knowledge bases ${ }^{25}$ to identify the key types of biological production workflows (i.e. metabolic engineering strategies ${ }^{26}$ ) that need to be modified for different space-based scenarios; (3) identify the supporting biomanufactory design elements within which these production workflows could be implemented ${ }^{27-29}$; and (4) identify the chassis organisms and other biological components $\mathrm{s}^{30-32}$ that will be required to compose the complete set for downstream engineering specifications. Systems designed from a minimal set of reliable parts, standard interconnects, and common controller languages also offer the best possible chance of characterized reliability under changing environmental conditions. Therefore, control of hardware and wetware should be augmented through the design and operation of software support. We see a fundamental effort in SBE as the amalgamation of space-driven hardware, software, and wetware that follows a synthetic biology DBTL cycle ${ }^{33}$.

The foundation of new SBE performance metrics that guide the design phase of the DBTL cycle must be augmented with additional downstream efforts in the build and test phases to (1) develop a process design framework that takes in specific production needs in amounts/time over acceptable ranges under the constraints expected across different offworld scenarios; (2) create the biological, process, and mission design software platforms to allow sophisticated DBTL, risk assessment, and mission choice support; (3) create the sensor/controller sets that will allow real-time optimization of biological production workflows; and (4) develop the online process controller framework that coordinates reactor conditions and inter-reactor flows to optimize reliable production across all units within acceptable ranges with minimal power and risk. The realization of this SBE DBTL cycle depends on the integration of such benchmark models and modeling standards. These benchmarks describe the dynamics of all SBE processes and relate to the SBE metrics in the design phase from which optimization can be carried out in the learn phase.

DBTL cycles within the scope of SBE must prepare for both ground- and flight-based system operations. Ground-based developments must prioritize designs that meet the requirements for flight-based testing, during which system behaviors may be better characterized in unique environments such as those offered in micro- and zero-gravity. For instance, a biological nitrogen-fixing system on earth must at least be designed to meet the mass and volumetric constraints required for validated 
ground-based simulators of microgravity, GCR, other physical stressors. Meeting certain requirements for time, power, and substrate usage is essential for any degree of long-term operation. This allows for the in-flight testing of bioreactors test-bedded on-earth that can more directly measure the effects micro-gravity, radiation and other stressors on the bioprocessing system. A combination of ground- and flight- based tests are required for the development of functional and robust space biosystems.

\section{Development of Means for SBE Flight}

Deployment of SBE platforms as mission critical elements will likely be reserved for longer duration human exploration missions such as those in the Artemis or Mars programs ${ }^{10}$. These future programs are still in the concept and planning stage in development, but will certainly be composed of a myriad of technologies that range in degree of flight-readiness as standardized by NASA's Technology Readiness Level ${ }^{34}$ (TRL, used to rate the maturity of a given technology during the acquisition phase of a program). Recent updates in NASA's definitions of and best-practices for applying the TRL paradigm led

\begin{tabular}{|c|c|c|c|c|c|}
\hline Platform & Volume & Power & Op. Lifetime & Temperature & Air Comp. \\
\hline $\begin{array}{l}\text { CubeSat } \\
\text { PocketQube }\end{array}$ & $\begin{array}{l}0.0187 \mathrm{~m}^{3} \\
0.000125 \mathrm{~m}^{3}\end{array}$ & $\begin{array}{l}20-45 \mathrm{~W} \\
\text { Variable }\end{array}$ & $\begin{array}{l}\sim 20 \text { years } \\
\sim 5 \text { years }\end{array}$ & $\begin{array}{l}\text { Requires heating unit } \\
\text { within constraints }\end{array}$ & Self-contained \\
\hline Bioculture System & Not stated & $140 \mathrm{~W}$ & $\sim 60$ days & $\begin{array}{l}37-45^{\circ} \mathrm{C} \text { in main } \\
\text { chamber, ambient to } \\
5^{\circ} \mathrm{C} \text { in cooling chamber }\end{array}$ & $\begin{array}{l}\text { Self-contained } \\
\text { medical grade gas }\end{array}$ \\
\hline $\begin{array}{l}\text { WetLab-2 } \\
\text { (SmartCycler) }\end{array}$ & $235.97 \mathrm{~m}^{3}$ & $350 \mathrm{~W}$ & $\begin{array}{l}\text { Extractions }<3 \mathrm{hrs} \text {, } \\
\text { no lifetime stated }\end{array}$ & $50-95^{\circ} \mathrm{C}$ & \\
\hline $\begin{array}{l}\text { Rodent Habitat } \\
\text { Hardware System }\end{array}$ & $0.019 \mathrm{~m}^{3}$ & Not stated & $\sim 30$ day experiments & & \\
\hline $\begin{array}{l}\text { Compact Science } \\
\text { Experiment Module }\end{array}$ & $0.0015 \mathrm{~m}^{3}$ & $3.2 \mathrm{~W}$ & $>1$ month experiments & & None, reliant on \\
\hline $\begin{array}{l}\text { Vegetable } \\
\text { Production } \\
\text { System (Veggie) }\end{array}$ & $\begin{array}{l}0.48 \mathrm{~m}^{3} \\
\text { growth area }\end{array}$ & & $\begin{array}{l}>12 \text { day experiments, } \\
\text { can replace crops }\end{array}$ & $\begin{array}{l}\text { Ambient temp, no } \\
\text { heating module }\end{array}$ & cabin air system \\
\hline $\begin{array}{l}\text { Advanced Plant } \\
\text { Habitat (APH) }\end{array}$ & $\begin{array}{l}889.44 \mathrm{~m}^{3} \\
\text { growth area }\end{array}$ & & $\sim 1$ year & $18-30^{\circ} \mathrm{C}$ & $\begin{array}{l}\text { Self-contained } \\
\text { gas supply }\end{array}$ \\
\hline Spectrum & $\begin{array}{l}10 \times 12.7 \mathrm{~cm} \\
\text { internal area }\end{array}$ & Not stated & 12 day experiments & $18-37^{\circ} \mathrm{C}$ & $\begin{array}{l}\text { None, reliant on } \\
\text { cabin air comp } \\
60 \mathrm{M} \text { variant can }\end{array}$ \\
\hline BRIC-60 & $11.03 \mathrm{~m}^{3}$ & & & & $\begin{array}{l}\text { draw from an external } \\
\text { gas tank }\end{array}$ \\
\hline BRIC-100 & $38.78 \mathrm{~m}^{3}$ & & 12 day expe & & Self-contained gas \\
\hline BRIC-100VC & $16.33 \mathrm{~m}^{3}$ & Unpowered & 4.5 months & Ambient temp, & canister of designated \\
\hline $\begin{array}{l}\text { KSC Fixation } \\
\text { Tubes (KFTs) }\end{array}$ & $0.2387 \mathrm{~m}^{3}$ & & 67 days & & $\begin{array}{l}\text { Airtight, reliant on } \\
\text { cabin air comp }\end{array}$ \\
\hline miniPCR & $0.00066 \mathrm{~m}^{3}$ & $65 \mathrm{~W}$ & $\sim 2$ year & $<120^{\circ} \mathrm{C}$ & \\
\hline $\begin{array}{l}\text { Group Activation } \\
\text { Pack-Fluid Processing } \\
\text { Apparatus (GAP-FPA) }\end{array}$ & $\begin{array}{l}\text { Eight } 6.5 \mathrm{~cm}^{3} \\
\text { test tubes }\end{array}$ & $\begin{array}{l}\text { Unpowered } \\
\text { for manual }\end{array}$ & & $4-37^{\circ} \mathrm{C}$ & \\
\hline $\begin{array}{l}\text { Multi-use Variable-g } \\
\text { Platform (MVP) }\end{array}$ & $\begin{array}{l}\text { Twelve } 800 \mathrm{~cm}^{3} \\
\text { modules }\end{array}$ & Not stated & Not stated & $14-40^{\circ} \mathrm{C}$ & Airtight, reliant on \\
\hline MinION & $0.0796 \mathrm{~m}^{3}$ & $5 \mathrm{~W}$ & $\sim 1$ year & $\begin{array}{l}\text { Ambient temp, no } \\
\text { heating module }\end{array}$ & \\
\hline $\begin{array}{l}\text { Perseverance } \\
\text { (MOXIE) }\end{array}$ & $0.017 \mathrm{~m}^{3}$ & $300 \mathrm{~W}$ & $\sim 2$ years & $\begin{array}{l}800^{\circ} \mathrm{C} \text { operational } \\
-60^{\circ} \mathrm{C} \text { ambient }\end{array}$ & $\begin{array}{l}\mathrm{CO}_{2} \text { input } \\
\mathrm{CH}_{4} \text { output }\end{array}$ \\
\hline $\begin{array}{l}\text { Gateway } \\
\text { (HALO) }\end{array}$ & $\begin{array}{l}>125 \mathrm{~m}^{3} \text { planned } \\
\text { internal volume }\end{array}$ & $\sim 60 \mathrm{~kW}$ & $>2$ years & $\sim 18^{\circ} \mathrm{C}$ & Pressurized cabin air \\
\hline $\begin{array}{l}\text { Mars Hab } \\
\text { (6 Crew) }\end{array}$ & $300 \mathrm{~m}^{3}$ & $\sim 100 \mathrm{~kW}$ & $\begin{array}{l}600 \text { day nominal, } \\
619 \text { day maximum }\end{array}$ & $\sim 18^{\circ} \mathrm{C}$ & Pressurized cabin air \\
\hline
\end{tabular}

Table 1. Constraints on past and current experimental platforms including Small Satellites (light blue), Space Stations (medium blue), Rovers (dark blue), planned Lunar Habitation (light red), and Martian Habitation (red). The shade of color darkens with increasing complexity and cost. The specific sources can be found in the SI. 
to the standardization and merging of exit criteria between hardware and software systems ${ }^{35}$. However, the TRL concept as it relates to SBE must be further expanded to include definitions and exit criteria for 'wetware' in addition and in relationship to hardware and software elements.

Deployment of SBE is space requires a level of rigor in technology acceptance that is of a different order than most earth-based systems because mission failures are exceptionally costly and difficult to recover from. The missions into which SBE processes will integrate are hugely complicated and as noted above will be interdependent in complex ways. Thus while low levels TRLs can be reach through unit testing in modest formats both on earth and limited flight chasses, the integrated nature of the bioprocess control and engineering will require integration testing even at the TRL 4 and 5 levels $^{35}$. To meet acceptance at TRL 6 and beyond will require long term planning realistic integration and deployment testing with actual sophisticated space missions and their logistics.

Even at low TRLs, research on the timescales needed to validate extended-use systems as would be leveraged on extendedstay forward deployment such as Martian or lunar missions are not possible given the current ISS capabilities and constraints. Constraints in astronaut time and limitations in hardware designed for shorter experiments prevent testing times comparable to long duration missions. Table 1 outlines a number of constraints on past and current experimental platforms and provides some basis for constraints of future systems (Fig. 1d). Here we note that extended multigenerational studies, especially in microbiology, can be difficult with some of the operational lifetimes. ${ }^{36}$. Volume is also constrained, and available space is broken up into segmented rack testbeds and independent machines, which can prevent aspects of a system from interacting with each other (Table 1). Much of the testing hardware on the ISS is designed for front-end processing and basic science, and many experiments in microbial observation ${ }^{37,38}$, hybrid life support ${ }^{39}$, antibiotic response ${ }^{40}$, and more all require returning samples to Earth for efficient processing, limiting the end-product downstream analysis and use as feedstocks for other integrated processes, as is needed to advance TRL beyond 6. This also cuts down on the ability to run DBTL diagnostics and SBE performance metrics on the system in toto as recyclability and sustainability are reliant on those end-products, and supportability if the processing is often reliant on Earth resources. Though much of the potential testing: $\mathrm{PCR}^{41}$, imaging ${ }^{42}$, and DNA sequencing ${ }^{43,44}$ is possible with current miniaturized ISS modules, it may not all be at the scale needed for future experiments, and there may be gaps in capability as the field matures. Improved in situ data analysis through development of new, high-throughput instruments could help suture those gaps ${ }^{45}$ and allow better metricization of whole systems under these new performance paradigms.

Lunar and Martian gravity can potentially have distinct biological effects compared to Earth gravity, resource composition, and radiation profile - and the ISS has only a limited volume in which to simulate them ${ }^{46}$. Additionally, both ambient environmental and target temperature windows span an extensive range across extraterrestrial environments, as do gas compositions, making representative testing more difficult in growth and testing chambers (plant, animal, and microbial) without full environmental control (Table 1). ECLSS systems for large-scale plant science requisite for advancing TRL for downstream lunar and Martian missions also require larger volume bounding boxes than is currently provided on the ISS ${ }^{47}$. Here we note the trade-offs with the tight volume and power stores on board. Smaller satellite modules can get technologies off the ground to advance TRL ${ }^{48-50}$, but feature even greater size handicaps, and may prevent testing at the integrated, factory level in the DBTL cycle ${ }^{51,52}$. Scientific instruments and modules on rovers have been geared primarily for exploration and observation, not technology validation. Dedicated rovers or simply landing SBE payloads onto extraterrestrial sites, SBE-ready orbiters, and Artemis operations as a stepping-stone to Mars can all demonstrate technology within a representative context and stand as some of the premier testbeds to "flight qualify" SBE prototypes ${ }^{34}$. In situ testing is key to the proposed SBE performance metrics: it forces technology and bioprocesses into accurate, integrated environments, and provides better confidence under radiation, microgravity, and isolation.

\section{Training of SBE Minds}

Maturation of space bioprocess engineering requires specialization of the training needed to produce the next generation of spacefaring scientists, engineers, astronauts, policy makers, and support staff ${ }^{53}$. Lessons learned from the Space Transportation System (STS) era led to calls for an increase in Science-Technology-Engineering-Mathematics (STEM) educational programs ${ }^{54}$ beginning in secondary schools ${ }^{55}$ and propagating to novel astronautics-based undergraduate ${ }^{56}$ and graduate programs ${ }^{57}$, and to the establishment of specialty space research centers ${ }^{58}$ focused on technology transfer ${ }^{59}$. The calls for workforce development were repeated just prior to the collapse of the STS program, noting the dangers likely to arise from the lack of educational and training resources for those entering the space industry. ${ }^{60}$. Such a risk as described is especially poignant in the case of space-based biotechnologies given that mature technologies are far fewer, the new applications more futuristic, and the disciplines are not well represented in the traditional physics and engineering curricula. The Universities Space Research Association (USRA) lists 114 institutions with Space Technologies/Science academic programs while recent accounting of bioastronautics programs numbers $36^{61}$. However, the intersection between these lists yields only 22 schools. Given that US News names 250 world schools that have tagged themselves with Space Science programs, only $~ 8 \%$ of these are 


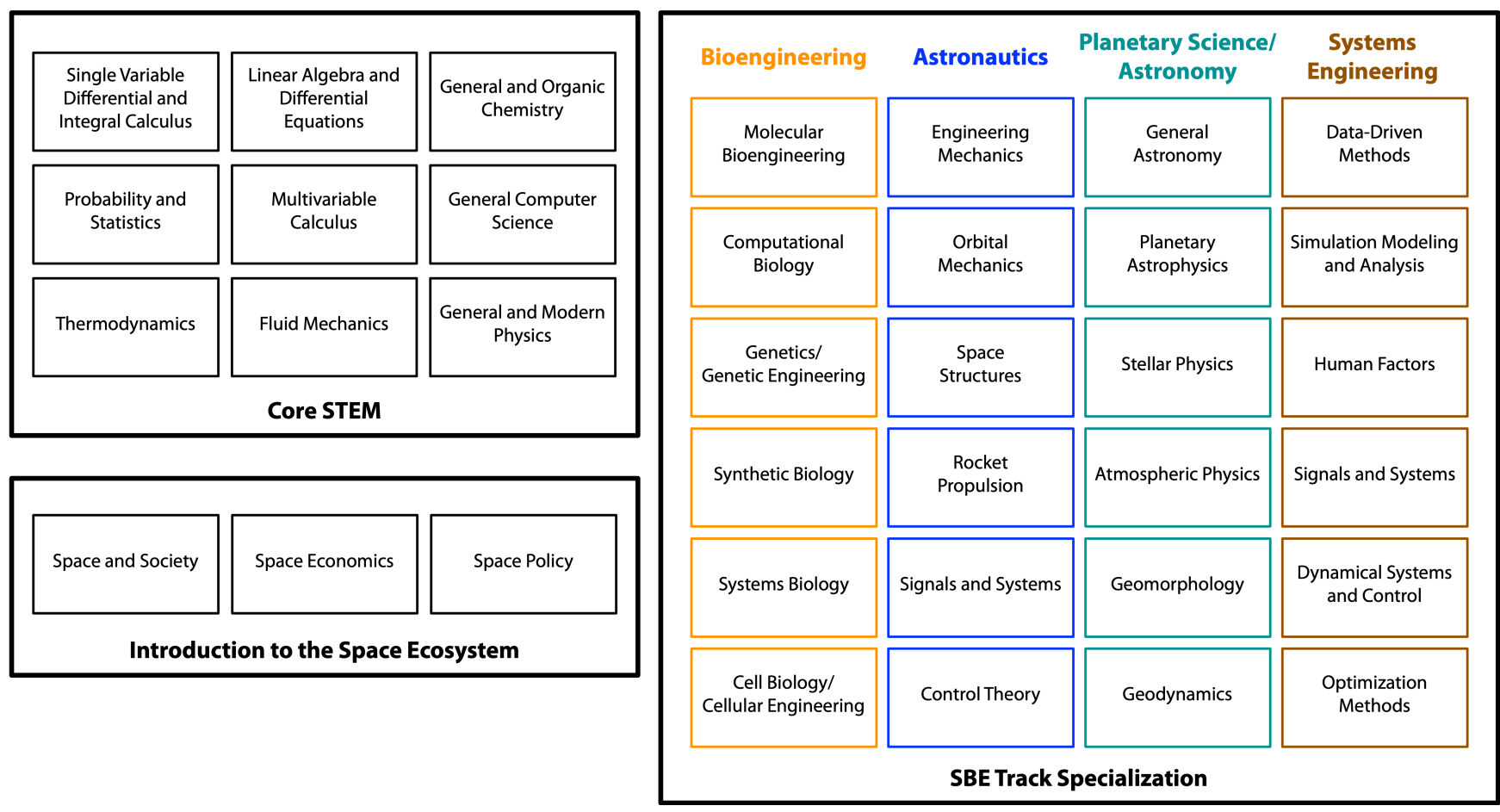

Figure 3. Conceptual undergraduate SBE program.

currently offering bioastronautics specialization - demonstrating that efforts that integrate human performance, life support and bioengineering are under-served. Furthermore, the bioastronautics programs such as those offered by schools like Harvard-MIT, University of Colorado Boulder, and Baylor University are not focused on biomanufacturing aspects that underlie $\mathrm{SBE}^{62}$.

Academia must be prepared to capitalize on the opportunities of future SBE applications starting with either the creation of new and interdisciplinary programs or by assembling those from related disciplines (Fig. 1a). Because scientific and mathematical core courses are relatively standard across SBE-related disciplines, an effective foundation of technical skills could be easily constructed from the shared curriculum (Fig. 3). From there, specific SBE-driven training can be offered in (1) effects of space on plant and microbes; (2) process design for low gravity/high radiation; (3) management and storage of biological materials in space based operations; (4) low energy/low mass bioreactor/bioprocessor design; (5) integrated biological systems engineering; (6) biological mission planning and logistics; (7) risk and uncertainty management; (8) containment and environmental impact of biological escape, films, corrosion and cleanup; and (9) ethics of cultivation and deployment. While the logistics for organizing such pathways for formal SBE training are non-trivial within the academic machine, we note that nearly all schools listed by USRA offer the component programs in bioengineering, planetary science or astronomy, and electrical or systems engineering. Since the courses for such engineering programs are standardized ${ }^{63}$, it stands to reason that establishing focused SBE programs can begin by collecting and highlighting course combinations. As programs grow, additional faculty with SBE-driven research can be sourced. Such openings offer a much needed opportunity to address systemic issues of diversity, equity, and inclusion both within SBE-based academia and the industrial space community at large ${ }^{64}$.

\section{Moving Forward}

Making progress on the program above requires scientists, engineers, and policy experts to work together to verify, open, and update campaign specifications. The science requires scientists from multiple disciplines spanning biological and space systems engineering that require a degree of modularity, small footprints, and robustness not found elsewhere. Additionally, bioprocess and biological engineering must be applied to the building of cross-compatible and scalable processing systems and optimized organisms within the confines of space reactor and product. Finally, coordination mission specialists are critical to deploy tests into space during the run-up and through crewed missions. We argue that such groundwork requires multidisciplinary centers that can build long term partnerships and understanding; train the workforce in this unique application space; and perform the large-scale, long-term science necessary to succeed. 


\section{Acknowledgements}

This material is based upon work supported by NASA under grant or cooperative agreement award number NNX17AJ31G.

\section{Authorship Contributions}

AJB, AM, JMH, APA conceived the concept based on the Center for the Utilization of Biological Engineering in Space (CUBES). DH led the graphics effort with assistance from AJB. GM, IL, NJHA, AAM, AM, and APA contributed to research and analyses. All authors wrote and edited the manuscript.

\section{Competing Interests}

The authors declare that they have no conflicts of interest.

\section{References}

1. Menezes, A. A., Cumbers, J., Hogan, J. A. \& Arkin, A. P. Towards synthetic biological approaches to resource utilization on space missions, vol. 12 (The Royal Society, 2015).

2. Nangle, S. N. et al. The case for biotech on Mars. Nat. Biotechnol. 38, 401-407 (2020).

3. Young, L. R. \& Sutton, J. P. Handbook of Bioastronautics (Springer, 2020).

4. Whitmore, M., Boyer, J. \& Holubec, K. NASA-STD-3001, Space Flight Human-System Standard and the Human Integration Design Handbook. In Industrial and Systems Engineering Research Conference (2012).

5. Hoffman, S. J. \& Kaplan, D. I. Human exploration of Mars: the reference mission of the NASA Mars exploration study team, vol. 6107 (National Aeronautics and Space Administration, Lyndon B. Johnson Space Center, 1997).

6. Drake, B. G., Hoffman, S. J. \& Beaty, D. W. Human exploration of Mars, design reference architecture 5.0. In Aerospace Conference, 2010 IEEE, 1-24 (IEEE, 2010).

7. Fridlund, M. \& Lammer, H. The astrobiology habitability primer. Astrobiology 10, 1-4 (2010).

8. Talbert, T. \& Green, M. Space Technology Grand Challenges (2010).

9. Menezes, A. A., Montague, M. G., Cumbers, J., Hogan, J. A. \& Arkin, A. P. Grand challenges in space synthetic biology. J. The Royal Soc. Interface 12, 20150803, DOI: 10.1098/rsif.2015.0803 (2015).

10. Berliner, A. J. et al. Towards a Biomanufactory on Mars. Front. Astron. Space Sci. 8, 120, DOI: 10.3389/fspas.2021.711550 (2021).

11. Cestellos-Blanco, S. et al. Production of PHB From CO2-Derived Acetate With Minimal Processing Assessed for Space Biomanufacturing. Front. Microbiol. 12, 2126, DOI: 10.3389/fmicb.2021.700010 (2021).

12. Langenfeld, N. J. et al. Optimizing Nitrogen Fixation and Recycling for Food Production in Regenerative Life Support Systems. Front. Astron. Space Sci. 8, 105, DOI: 10.3389/fspas.2021.699688 (2021).

13. Rapp, D. Mars ISRU technology. In Use of Extraterrestrial Resources for Human Space Missions to Moon or Mars, 31-90 (Springer, 2013).

14. Werkheiser, N. In-space manufacturing: pioneering a sustainable path to Mars. (2015).

15. Cannon, K. M. \& Britt, D. T. Feeding one million people on Mars. New Space 7, 245-254 (2019).

16. McNulty, M. J. et al. Molecular Pharming to Support Human Life on the Moon, Mars, and Beyond. Critical Rev. Biotechnol. 0, 1-16, DOI: 10.20944/PREPRINTS202009.0086.V1 (2020).

17. Poughon, L., Farges, B., Dussap, C. G., Godia, F. \& Lasseur, C. Simulation of the MELiSSA closed loop system as a tool to define its integration strategy. Adv. Space Res. 44, 1392-1403, DOI: https://doi.org/10.1016/j.asr.2009.07.021 (2009).

18. NASA. NASA's Journey to Mars: Pioneering Next Steps in Space Exploration. Tech. Rep. (2015). DOI: NP-2015-08-2018-HQ.

19. A Midterm Assessment of Implementation of the Decadal Survey on Life and Physical Sciences Research at NASA. Tech. Rep., National Academy of Sciences (2018).

20. DOE Announces New $\$ 60$ Million Investment to Increase Energy Efficiency in Manufacturing (2021).

21. Marshall Space Flight Center Space Systems. Tech. Rep., National Aeronautics and Space Administration, Marshall Space Flight Center, Huntsville, AL (2011). DOI: NP-2011-05-051-MSFC8\T1\textendash477491b. 
22. Chapline, G. \& Sullivan, S. Systems engineering for Lifecycle of Complex Systems. Eng. Innov. (NASA) (2010).

23. Cohen, J. The crucial role of CS in systems and synthetic biology. Commun. ACM 51, 15-18 (2008).

24. Averesch, N. J. H. Choice of Microbial System for In-Situ Resource Utilization on Mars. Front. Astron. Space Sci. 8, 116, DOI: 10.3389/fspas.2021.700370 (2021).

25. Arkin, A. P. et al. KBase: the United States department of energy systems biology knowledgebase. Nat. biotechnology 36, 566-569 (2018).

26. Lucks, J. B., Qi, L., Whitaker, W. R. \& Arkin, A. P. Toward scalable parts families for predictable design of biological circuits. Curr. opinion microbiology 11, 567-573 (2008).

27. Carbonell, P. Getting on the Path to Engineering Biology. In Metabolic Pathway Design, 3-10 (Springer, 2019).

28. Appleton, E., Madsen, C., Roehner, N. \& Densmore, D. Design automation in synthetic biology. Cold Spring Harb. perspectives biology 9 , a023978 (2017).

29. Goñi-Moreno, A. et al. An implementation-focused bio/algorithmic workflow for synthetic biology. ACS synthetic biology 5, 1127-1135 (2016).

30. Shetty, R. P., Endy, D. \& Knight, T. F. Engineering BioBrick vectors from BioBrick parts. J. biological engineering 2, $1-12$ (2008).

31. Canton, B., Labno, A. \& Endy, D. Refinement and standardization of synthetic biological parts and devices. Nat. biotechnology 26, 787-793 (2008).

32. Cox, R. S. et al. Synthetic biology open language (SBOL) version 2.2. 0. J. integrative bioinformatics 15 (2018).

33. Cheng, A. A. \& Lu, T. K. Synthetic biology: an emerging engineering discipline. Annu. review biomedical engineering 14, 155-178 (2012).

34. Mankins, J. C. Technology readiness levels. White Pap. April 6, 1995 (1995).

35. Hirshorn, S. \& Jefferies, S. Final report of the NASA Technology Readiness Assessment (TRA) study team. (2016).

36. Castro, S. L., Smith, D. J. \& Ott, M. Researcher's Guide to: International Space Station Microbial Research. Tech. Rep., National Aeronautics and Space Administration, Johnson Space Center, Houston, TX (2014).

37. Khodadad, C. L. M. et al. Microbiological and Nutritional Analysis of Lettuce Crops Grown on the International Space Station. Front. Plant Sci. 11, 199 (2020).

38. Burton, A. S. et al. Off Earth Identification of Bacterial Populations Using 16S rDNA Nanopore Sequencing. Genes 11, DOI: 10.3390/genes11010076 (2020).

39. Keppler, J. et al. The final configuration of the algae-based ISS experiment PBR@ LSR. In 48th International Conference on Environmental Systems (ICES, Albuquerque, NM, 2018).

40. Aunins, T. R. et al. Spaceflight Modifies Escherichia coli Gene Expression in Response to Antibiotic Exposure and Reveals Role of Oxidative Stress Response. Front. Microbiol. 9, 310, DOI: 10.3389/fmicb.2018.00310 (2018).

41. Boguraev, A.-S. et al. Successful amplification of DNA aboard the International Space Station. npj Microgravity 3, 26, DOI: 10.1038/s41526-017-0033-9 (2017).

42. Levine, H. G. \& Flowers, D. A. Spectrum. Tech. Rep., NASA Ames Research Center, Kennedy Space Center, Merritt Island, Florida (2019).

43. McIntyre, A. B. R. et al. Nanopore sequencing in microgravity. npj Microgravity 2, 1-9 (2016).

44. Joannès, J. Feasibility Study of a DNA-Sequencing Cubesat Satellite. J. Br. Interplanet. Soc. 70, 287-299 (2017).

45. Karouia, F., Peyvan, K. \& Pohorille, A. Toward biotechnology in space: High-throughput instruments for in situ biological research beyond Earth. Biotechnol. advances 35, 905-932 (2017).

46. Zavaleta, J., Iyer, J., Mhatre, S., Dolling-Boreham, R. \& Bhattacharya, S. An Automated Behavioral Analysis of Drosophila Melanogaster. In Annual Meeting of the American Society for Gravitational and Space Research (2019).

47. Suffredini, M. T. Reference guide to the international space station. Tech. Rep., National Aeronautics and Space Administration, Johnson Space Center, Houston, TX (2014). DOI: NP-2015-05-022-JSC.

48. Marzioli, P. et al. CultCube: Experiments in autonomous in-orbit cultivation on-board a 12-Units CubeSat platform. Life Sci. Space Res. 25, 42-52, DOI: https://doi.org/10.1016/j.lssr.2020.02.005 (2020). 
49. Luna, A., Meisel, J., Hsu, K., Russi, S. \& Fernandez, D. Protein structural changes on a CubeSat under rocket acceleration profile. npj Microgravity 6, 12, DOI: 10.1038/s41526-020-0102-3 (2020).

50. Santoni, F. et al. GreenCube: microgreens cultivation and growth monitoring on-board a 3 U CubeSat. In 2020 IEEE 7th International Workshop on Metrology for AeroSpace (MetroAeroSpace), 130-135, DOI: 10.1109/MetroAeroSpace48742. 2020.9160063 (2020).

51. Cubesat 101: basic concepts and processes for first-time CubeSat developers. Tech. Rep. (2017).

52. Johnstone, A. CubeSat design specification (1U-12U) rev 14 CP-CDS-R14. Tech. Rep., California Polytechnic State University, San Luis Obispo, CA (2020). DOI: CP-CDS-R14.

53. Hurlbert, K. et al. Human Health, Life Support and Habitation Systems Technology Area 06. NASA Sp. Technol. Roadmaps (2012).

54. Farmer, T. A STEM Brainstorm at NASA. Tech. Connect. Educ. Careers (J1) 84, 42-43 (2009).

55. Engle, H. A. \& Christensen, D. L. Identification and evaluation of educational uses and users for the STS. Educational planning for utilization of space shuttle ED-PLUSS. (1974).

56. Brodsky, R. F. The Time Has Come for the BS in Astronautical Engineering. Eng. Educ. 76, 149-152 (1985).

57. Sonnenfeld, G. NASA Space Biology Research Associate Program for the 21st Century. (1999).

58. Mclntire, L. V. \& Rudolph, F. B. NASA Specialized Center of Research and Training (NSCORT) in Gravitational Biology. (1996).

59. Fletcher, L. S. \& Page, R. H. Technology transfer: The key to successful space engineering education. Acta Astronaut. 29 , 141-146, DOI: https://doi.org/10.1016/0094-5765(93)90032-R (1993).

60. Gruntman, M. The Time for Academic Departments in Astronautical Engineering. In AIAA SPACE 2007 Conference \& Exposition, DOI: 10.2514/6.2007-6042 (2007).

61. Young, L. R. \& Natapoff, A. The Harvard-MIT PHD Program in Bioastronautics. Life Space for Life on Earth 553, 90 (2008).

62. Klaus, D. M. Incorporating Bioastronautics into an Engineering Curriculum (44th International Conference on Environmental Systems, 2014).

63. Criteria for Accrediting Engineering Programs, 2020 - 2021 I ABET (2021).

64. National Aeronautics And Space Administration (NASA) Model Equal Employment Opportunity Program Status Report: FY 2019 . Tech. Rep., NASA (2020). 